\title{
Nonlinear lensing mechanisms in a cloud of cold atoms
}

\author{
G. Labeyrie ${ }^{1}$, G.L. Gattobigio ${ }^{1,2}$, T. Chanelière ${ }^{1,3}$, G.L. Lippi ${ }^{1}$, T. Ackemann ${ }^{4}$, and R. Kaiser ${ }^{1, a}$ \\ 1 Institut Non Linéaire de Nice, UMR 6618 CNRS, 1361 route des Lucioles, 06560 Valbonne, France \\ 2 Dipartimento di Fisica dell'Università di Ferrara and INFN-Sezione di Ferrara, 44100 Ferrara, Italy \\ 3 Georgia Institute of Technology, Atlanta, Georgia 30332, USA \\ 4 SUPA, Department of Physics, University of Strathclyde, Glasgow G4 ONG, UK
}

Received 18 July 2006 / Received in final form 5 September 2006

Published online 20 October 2006 - (c) EDP Sciences, Società Italiana di Fisica, Springer-Verlag 2006

\begin{abstract}
We present an experimental study of nonlinear lensing of near-resonant light by a cloud of laser-cooled rubidium atoms, specifically aimed at understanding the role of the interaction time between the light and the atomic vapor. We identify four different nonlinear mechanisms, each associated with a different time constant: electronic nonlinearity, Zeeman optical pumping, hyperfine optical pumping and radiation pressure. Our observations can be quite accurately reproduced using a simple rate equation model which allows for a straightforward discussion of the various effects. The results are important for planning more refined experiments on transverse nonlinear optics and self-organization in samples of cold atoms.
\end{abstract}

PACS. 42.65.Jx Beam trapping, self-focusing and defocusing; self-phase modulation - 32.80.Pj Optical cooling of atoms; trapping - 42.65.Sf Dynamics of nonlinear optical systems; optical instabilities, optical chaos and complexity, and optical spatio-temporal dynamics

\section{Introduction}

The reshaping of the profile of an intense laser beam during the propagation in a nonlinear (NL) medium is a subject of active research since the advent of the laser. It covers subjects like self-focussing (and self-defocussing) [1-5], solitons [3], wave-mixing [6-8], filamentation [9-13] and the spontaneous appearance of optical vortices $[14,15]$. Nonlinear propagation has been studied in many materials, ranging from atomic vapors (cf., e.g. [16] for a review) to solids, semiconducting materials, liquid crystals, photorefractive crystals, etc. (a most recent review can be found in Ref. [17]). We present in this paper a time-resolved study of self-lensing in a laser-cooled atomic cloud.

Besides the interest in self-lensing per se as the basic form of self-action of light involving the transverse degrees of freedom, its understanding is also regarded as the prerequisite for planning experiments on more complex forms of transverse self-organization like the formation of solitons and pattern formation in the presence of feedback (e.g., [16-19]). In a broader context, it is expected to be also relevant for light transport in nonlinear random media, e.g., light localization [20] or random lasing [21] using cold atomic vapors.

Compared to photorefractive media, where the nonlinearity is very high (cf. [22] for a recent review) but where modeling from first principles is impossible, cold atomic

\footnotetext{
a e-mail: Robin.Kaiser@inln.cnrs.fr
}

samples offer an attractive alternative since the atomic structure is very well-known and controlled and can be closely described with a limited set of variables. In addition, the high degree of control intrinsic to atomic manipulation allows the investigator to introduce verifiable approximations whose degree of accuracy can be tested with good confidence levels. In hot vapors, the complexity of modeling the (potentially already complex) spatiotemporal dynamics will be greatly enhanced by the need to consider Doppler broadening and the interaction of the light with different velocity classes unless a very strong homogeneous broadening is introduced by addition of a buffer gas [16,23], which, in turn, reduces the nonlinear response. In cold atoms, this complication does not exist, at least in the early stages of the experiment (see Sect. 3.2.1). Last but not least, an additional ingredient intrinsic to cold vapors is the mechanical action of light on the atoms, which may rearrange themselves during the interaction to form structures which strengthen and modify the nonlinear propagation properties of the medium [24,25].

In a previous paper [26], we reported a first observation of self-lensing in a cold atomic sample. Since then, nonlinear beam focussing has been observed in a setup somewhat different from ours [27] and has been modeled [28]. Although a qualitative agreement could be found between observations and a simple two-level theory [26-28], the role of the interaction time in these experiments was not discussed in detail. However, some time dependence of the NL lensing signal was experimentally observed in 
reference [27], and mechanisms based on Zeeman optical pumping and radiation pressure were proposed as candidates to explain this behavior. The present paper aims at clarifying this point. By studying the influence of the duration of the atom-light interaction, we identify four different mechanisms for nonlinear lensing. Similar enhanced nonlinearities induced by mechanical action of light have been observed in four wave mixing experiments [29] and collective atomic recoil lasers [30].

The paper is organized as follows. We first briefly describe the experimental procedure and detection techniques (Sect. 2). We then discuss the various nonlinear mechanisms (Sect. 3) where we focus on the electronic nonlinearity (Sect. 3.1), the mechanical effect of light (Sect. 3.2.1) and hyperfine pumping (Sect. 3.2.2). Throughout the paper we compare the experimental results to simple analytical and numerical results to support our observations.

\section{Experiment: principle and set-up}

In self-lensing experiments, the propagation of a laser beam in a nonlinear medium is affected by the transverse profile of the light field itself. Typically, the inhomogeneous intensity profile $I(R)$ ( $R$ is the radial distance from the beam propagation axis, of coordinate $z$ ) imprints in the medium a transverse variation of the refractive index, i.e. a lens. Depending on the parameters, this lens can be "thin" (no propagation effects inside the medium) and yield self-focussing or defocussing of the beam, or "thick" and eventually lead to e.g. self-trapping of the beam (soliton) [31]. Associated with the modification of the refractive index, there usually is a transversely varying absorption profile which may produce attenuation and diffraction of the transmitted beam. One can easily express the phase shift $d \varphi$ and attenuation $d I$ experienced by the beam propagating through a layer of 2-level atoms at rest, of thickness $d z$ small enough such that the attenuation remains small $(d I / I \ll 1)$ :

$$
\begin{aligned}
d \varphi(R) & =\frac{2 \pi[n(R)-1] d z}{\lambda} \\
& =-\frac{3 \lambda^{2}}{2 \pi} \rho d z \frac{\delta / \Gamma}{1+4(\delta / \Gamma)^{2}+I(R) / I_{\text {sat }}}
\end{aligned}
$$

and

$$
d I(R)=-\frac{3 \lambda^{2}}{2 \pi} \rho d z \frac{I(R)}{1+4(\delta / \Gamma)^{2}+I(R) / I_{\text {sat }}} .
$$

In these expressions, $n$ is the refractive index of the atomic vapor, and $\rho$ its density. $\delta=\omega-\omega_{a t}$ is the frequency detuning of the light from the atomic resonance ( $\omega$ and $\omega_{a t}$ are the light and atomic angular frequencies respectively). In our case, this resonance corresponds to the $F=3 \rightarrow F^{\prime}=$ 4 dipole transition of the D2 line of rubidium, of wavelength $\lambda=780 \mathrm{~nm}$ and natural width $\Gamma / 2 \pi=5.9 \mathrm{MHz}$. The strength of the atom-light coupling is measured by the

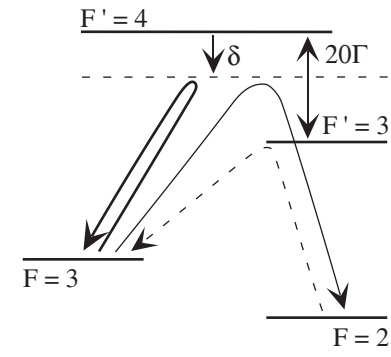

Fig. 1. Simplified level scheme of $\mathrm{Rb}^{85}$ (not drawn to scale). The atom-light interaction is mediated by the $F=3 \rightarrow F^{\prime}=4$ dipole transition of the $\mathrm{D} 2$ line of $\mathrm{Rb}^{85}$. The laser is detuned from this transition by $\delta$. The off-resonant excitation in the $F^{\prime}=3$ and $F^{\prime}=2$ states (not shown) causes the decay in the lowest hyperfine state $F=2$, where the atom is not coupled to the light (hyperfine pumping). The adjunction of a repumping laser (dashed arrow) suppresses hyperfine pumping.

saturation parameter $s=\left(I / I_{\text {sat }}\right) /\left[1+4(\delta / \Gamma)^{2}\right]$, where $I_{\text {sat }}=1.6 \mathrm{~mW} / \mathrm{cm}^{2}$ is the saturation intensity for the transition: for resonant light of intensity $I=I_{\text {sat }}$ yielding $s=1,25 \%$ of the atomic population is in the excited state. $b_{r e s}=\left(3 \lambda^{2} / 2 \pi\right) \rho d z$ is the on-resonance optical thickness of the layer, which appears in both expressions above.

Expression (1) corresponds to the well-known dispersive behavior of the refractive index, which is smaller than unity for $\delta>0$ and larger for $\delta<0$. In the linear regime $s \ll 1$, expression (2) yields the usual Beer-Lambert law with an exponentially decreasing transmission $T=e^{-b}$, where $b=b_{\text {res }} /\left[1+4(\delta / \Gamma)^{2}\right]$ is the optical thickness. In the nonlinear regime, two situations may occur; if the incident beam saturation is large $s>b$, the sample is partially "bleached" and the transmission is $T \approx 1-b /(1+s)$; in the opposite case, the beam is strongly attenuated upon passing through the sample (if $b>1$ ). The transversely inhomogeneous phase shift and attenuation described by equations (1) and (2) will respectively produce self-lensing and "self-diffraction" of the transmitted beam after further propagation.

Our rubidium atom is quite far from the ideal 2level system described above, as illustrated in Figure 1. First, the ground state $F=3$ consists of seven degenerate Zeeman-sublevels. In the models employed in the present paper, we will assume that the atoms in the vapor are evenly distributed among these sublevels and neglect Zeeman optical pumping (see however the discussion in Sect. 3). The effect of the Zeeman degeneracy is then to reduce the effective Clebsch-Gordan coefficient from 1 to $3 / 7$. Accordingly, the linear atomic polarizability is reduced by this factor, and the effective saturation intensity is increased to $3.7 \mathrm{~mW} / \mathrm{cm}^{2}$. Second, there are two other excited states accessible in the hyperfine structure multiplet, namely the $F^{\prime}=3$ and $F^{\prime}=2$ levels which are distant from the $F^{\prime}=4$ state by 20 and 30 natural widths respectively. Through off-resonant excitation of these levels, the atom can decay into the lower $F=2$ ground state where it is lost for the atom-light interaction (this hyperfine pumping process is represented by the thin 


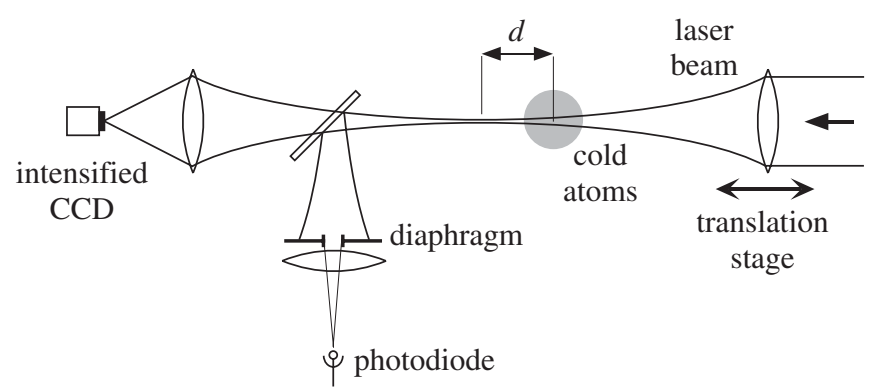

Fig. 2. Experimental set-up. A detuned, focussed laser beam is sent through the cloud of cold atoms ( $d$ is the distance separating the beam waist from the sample). The time-resolved far-field distribution of the transmitted beam is recorded with a gated CCD camera. The on-axis transmission can also be monitored using a photodiode.

arrow in Fig. 1). Hyperfine pumping can be efficiently suppressed by applying a "repumping" laser resonant with the $F=2 \rightarrow F^{\prime}=3$ transition (dashed arrow). Indeed, the presence of a repumping laser is essential for the operation of alkali magneto-optical traps (MOT).

To detect NL lensing effects, we employ an experimental configuration (see Fig. 2) similar to the traditional $z$ scan set-up [32]. A focussed laser beam is passed through the cloud of cold atoms. The beam waist of $1 / e^{2}$ radius $w_{0}$ is placed at a controlled distance $d$ from the medium, yielding a beam size $w$ inside the sample. The light polarization is linear. The on-axis transmitted intensity in the far-field is measured by placing a photodiode behind a diaphragm. To obtain spatially and time-resolved information on the nonlinear lensing signal, we also image the far-field intensity distribution of the transmitted beam on a gated CCD (Hamamatsu ORCAII) placed in the focal plane of a lens.

Our nonlinear sample is a cloud of laser-cooled $\mathrm{Rb}^{85}$ atoms, produced in a magneto-optical trap. A detailed description of the set-up can be found in reference [33]. For the experimental data presented here, the cold cloud contains $5 \times 10^{9}$ atoms and has a roughly Gaussian density distribution of $4.6 \mathrm{~mm}$ FWHM. This yields a peak density $\rho_{0} \simeq 4.5 \times 10^{10} \mathrm{~cm}^{-3}$ and an optical thickness $b_{\text {res }} \approx 20$. The dilute atomic vapor is at a temperature of $40 \mu \mathrm{K}$, corresponding to a $\mathrm{rms}$ velocity (modulus) of $0.11 \mathrm{~m} / \mathrm{s}$. For all practical purposes, the Doppler broadening associated with the atomic velocity dispersion can be neglected in this experiment. As mentioned before, the atom-light coupling is realized via the $F=3 \rightarrow F^{\prime}=4$ dipole transition of the D2 line.

The typical experimental sequence consists of a trapping phase of $30 \mathrm{~ms}$, followed by a measurement phase. Prior to the measurement all trapping laser beams are shut down (excepted the repumping laser which is present in some of the experiments described in this paper), as well as the magnetic field gradient. This "dark" phase lasts roughly $2 \mathrm{~ms}$, a duration short enough so that the atoms do not move significantly and can be fully recaptured when the MOT is turned back on. In the middle of the dark phase, the near resonant probe beam is turned on for $1 \mathrm{~ms}$. An image of the far-field distribution of the transmitted beam is acquired at a variable time delay $\Delta t$ after the probe beam is switched on. The duration of the gate applied to the CCD is $1 \mu \mathrm{s}$, which sets the temporal resolution of the data presented in this paper. At the end of the dark phase, the MOT is turned on again and the whole sequence is repeated. The self-lensing signal is obtained after averaging over typically 150 cycles.

\section{Different mechanisms in self-lensing experiments}

In previous studies of self-lensing by cold atoms [26,27], the theoretical model used to interpret experimental findings was the saturable Kerr nonlinearity associated with a two-level atom at rest, which was assumed instantaneous at the time scale of the experiments. However, some time dependence of the NL lensing signal was experimentally observed in reference [27], and mechanisms based on Zeeman optical pumping and radiation pressure were proposed as candidates to explain this behavior.

Indeed, several mechanisms for self-lensing can simultaneously be present in the cold atomic cloud, each of them with its characteristic time constant.

(1) The fastest is the electronic nonlinearity (2-level atom at rest) whose response time is of the order of the excited state lifetime $1 / \Gamma \simeq 27 \mathrm{~ns}$ at most (for strong coupling $s \gg 1$, the response time is of the order of the inverse Rabi frequency and can be much shorter).

(2) As mentioned before, Zeeman optical pumping is possible due to the presence of degenerate Zeeman sublevels in the ground state. This may introduce a timeand intensity-dependent atomic polarizability because of the different Clebsch-Gordan coefficient associated with each Zeeman substate. The Zeeman pumping impact on self-lensing has been considered explicitly in the case of hot vapors [34], and suggested in recent work with cold atoms [27]. Although the scattering of a single photon is in principle sufficient to change the Zeeman state of an atom, to significantly pump a cloud of cold atoms generally requires the exchange of many photons. The NL mechanism associated with Zeeman optical pumping is expected to react with a time constant of many $\Gamma^{-1}[35]$.

(3) Another effect relies on the hyperfine pumping mechanism described in the previous section, which typically requires the scattering of several hundreds of photons and an interaction time of the order of $10 \mu \mathrm{s}$. Its effect is to deplete the population of the hyperfine ground state which interacts with the laser beam $(F=3$ in our case, see Fig. 1) and to create a time- and intensity-dependent effective atomic density inside the sample.

(4) Finally, as mentioned in reference [27], the radiation pressure exerted by the laser beam accelerates the atoms producing a time-and intensity-dependent Doppler shift. This effect acts typically over $100 \mu \mathrm{s}$ or slower. For even longer interaction times $(\simeq \mathrm{ms})$, the transverse atomic motion induced by the dipole force may also play a role (see discussion in Sect. 3.2.1). 

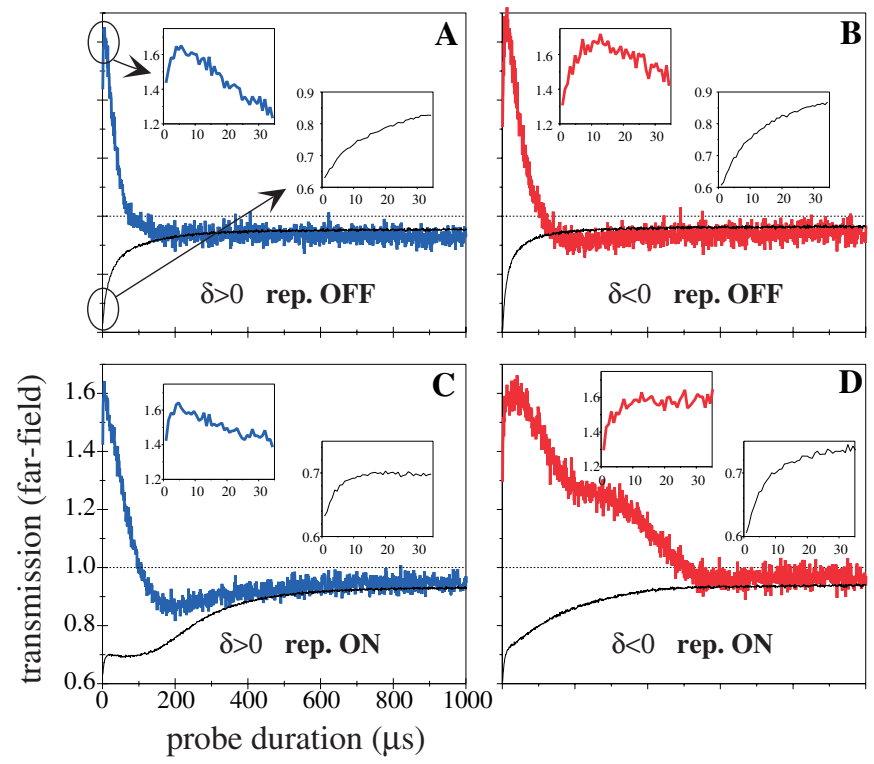

Fig. 3. (Color online) Experimental evidence for timedependent nonlinear mechanisms in cold atoms. We show timeresolved transmission signals in the far-field, either the total transmission (thin lines) or the on-axis transmission (bold lines, in color online), as measured by the photodiode of Figure 2. The inserts correspond to zooms of these curves during the first $35 \mu \mathrm{s}$ as shown by the arrows in quadrant $\mathrm{A}$. The left column correspond to $\delta>0$ and a beam waist placed before the sample (diverging input beam) while the right column is obtained for $\delta<0$ and a waist after the sample (converging input beam). The data of the top row is recorded without repumping laser, while it is present in the bottom row.

Figure 3 illustrates the existence of complex timedependent behaviors for the NL lensing signal. These farfield transmission curves are recorded using the photodiode + diaphragm set-up of Figure 2. In each quadrant of Figure 3, two curves are shown: the thin one corresponds to the total transmission (diaphragm fully open), while the bold, noisy one corresponds to the on-axis transmission (diaphragm diameter decreased down to approximately one half of the beam size at the diaphragm). The transmission is always defined as the ratio of the detected intensity in the presence of the cold atomic cloud to the intensity detected without atoms. The two inserts in each quadrant show zooms of the main curves during the first tens of $\mu \mathrm{s}$, as pointed by the arrows in quadrant $\mathrm{A}$. The left and right columns correspond to positive and negative detunings respectively $(|\delta|=2.3 \Gamma)$. When changing the sign of the detuning, one expect to change the sign of the nonlinear lens: this lens is positive (converging) for a positive detuning, and negative (diverging) for the opposite detuning [26]. To compensate for this sign change, we also change the waist position $d$ (see Fig. 2): for $\delta>0$ we focus the beam before the cloud, and for $\delta<0$ the waist is positioned after the cloud, at the same distance $(|d|=2.5 \mathrm{~mm})$. This allows us to compare similar self-lensing signatures, i.e. an increase of the on-axis transmission due to the collimation of the beam by the in- duced lens [26,27]. The laser power is $20 \mu \mathrm{W}$ and the beam waist $w_{0}=12 \mu \mathrm{m}$, as measured from the far-field imaging. The beam size in the atomic cloud is then $w \simeq 53 \mu \mathrm{m}$. The two rows of Figure 3 correspond to data recorded in the presence of a repumping beam (bottom row) or without it (top row). In our previous experimental study [26], no repumper was applied during the probe phase, while in the work of reference [27] the repumper was present (as well as the magnetic field gradient of the MOT, which is switched off in the present work).

In this experimental run, as well as for the data presented in the rest of the paper, the temporal resolution is of the order of $1 \mu \mathrm{s}$. Thus, we do not have access to the dynamical range of the electronic nonlinearity and can only measure its steady-state value. This is why all the on-axis transmission curves in Figure 3 start at values different from unity.

Let us now concentrate on the top row, where hyperfine pumping is present. We observe for red and blue detunings a rather similar result: the total transmission steadily increases from its initial value of $\approx 0.7$ towards 1 , with a time constant of the order of $15 \mu \mathrm{s}(13 \mu \mathrm{s}$ for $\delta<0$ and $17 \mu \mathrm{s}$ for $\delta>0$ ), while the on-axis transmission starts at roughly 1.65 and then decreases regularly towards the total transmission value. The on-axis transmission has dropped by half within $52 \mu$ s for blue detuning and $32 \mu$ s for red detuning.

A zoom of the on-axis transmission curves at short times (inserts) reveals a small initial increase up to a maximum reached between 5 and $10 \mu \mathrm{s}$, before the decrease takes place. This behavior is related to hyperfine pumping and will be discussed in Section 3.2.2.

We now address the case where the repumper beam is present during the probing phase (bottom row in Fig. 3), which suppresses hyperfine pumping. In this situation the picture changes markedly. First, the fast increase of the total transmissions is now replaced by a much slower evolution (time constant of $170 \mu$ s for red detuning). This proves that the fast evolution observed on the curves of the top row is due to hyperfine pumping. Second, the behavior of the total transmission is now strongly asymmetric with respect to detuning: for $\delta<0$ the transmission increases towards unity nearly exponentially while for $\delta>0$ it first slightly decreases to reach a minimum before increasing also. As will be discussed in Section 3.2.1, this red-blue asymmetry is a signature of the radiation pressure force exerted by the light beam on the atoms, an observation made possible by the use of cold atoms with a very narrow velocity distribution. The acceleration provided by the radiation pressure introduces, via the Doppler effect, a time-dependent effective light detuning which affects the atomic response. This Doppler shift always has the same sign (red shift), which breaks the symmetry between positive and negative laser detunings. This obviously has a deep impact on the NL lensing signal, as can be seen on quadrants $\mathrm{C}$ and D of Figure 3. We will analyze this effect in Section 3.2.1.

A zoom of the total transmission curves in $\mathrm{C}$ and D reveals a short $(\Delta t<10 \mu \mathrm{s})$ initial phase of rapidly 
increasing transmission, independent of the detuning sign. This observation is likely to be an indication of some Zeeman optical pumping. In this case, a similar behavior should also be observed in the absence of the repumper laser (top row in Fig. 3), but it can not be clearly identified since the increase of transmission due to hyperfine pumping occurs approximately with the same time constant. To understand the observed dynamics would require e.g. the knowledge of the initial population distribution among Zeeman substates when the probe beam is switched on. Since the overall effect is rather small, both on the total and on-axis transmissions, we will neglect Zeeman optical pumping in the rest of the paper.

In the following, we will concentrate on the NL response in the range from roughly $1 \mu \mathrm{s}$ to $1 \mathrm{~ms}$. Most of the dynamics in this range is due to radiation pressure and hyperfine pumping, as suggested by the data in Figure 3. These two mechanisms will thus be discussed in details in the rest of the paper. The results obtained for a short interaction time $(\Delta t<2 \mu \mathrm{s})$, which will be interpreted using the electronic nonlinearity alone, are detailed in the next section.

\subsection{Electronic nonlinearity}

In this section we discuss the characteristics of the nonlinearity for a 2-level atom at rest, and their impact in self-lensing experiments. To illustrate this situation, we performed a series of experiments with a low probe duration $\Delta t=2 \mu \mathrm{s}$, where the main contribution to the nonlinearity is electronic as discussed in the section before (and detailed in the following sections). The laser beam is focussed at a large distance $d=13 \mathrm{~cm}$ before the atomic cloud, with a waist size $w_{0}=47 \mu \mathrm{m}$. Because of this, the beam radius inside the sample is large $w \approx 700 \mu \mathrm{m}$. Under this conditions the self-lensing is fairly weak (see below) and the theoretical treatment we propose best justified.

The far-field images are recorded with the camera as described above. From these, we estimate the effective focal length of the vapor using the following procedure: we fit the far-field profile of the transmitted laser beam to a Gaussian, and from the obtained angular width compute the focal length using the conjugation formulae of Gaussian optics. The experimental data, shown in Figure 4, are obtained for two different light detunings: $\delta=+3 \Gamma$ (solid circles) and $\delta=+5 \Gamma$ (open circles). The focal length is plotted versus input power (note the logarithmic scales).

We observe that self-focusing is stronger for smaller detuning, which is explained by the higher index of refraction (see Eq. (1)). As a function of power, the focal length first decreases, then reaches a minimum and increases afterwards. The minimum is reached later for the larger detuning. A quick estimate shows that the minima correspond roughly to a saturation $s=1$ (arrows). This provides a starting point for the theoretical analysis.

Figure 5 shows how the transverse profile of the refractive index given by equation (1) evolves with increasing laser power. Two regimes can be identified: the "Kerr" and

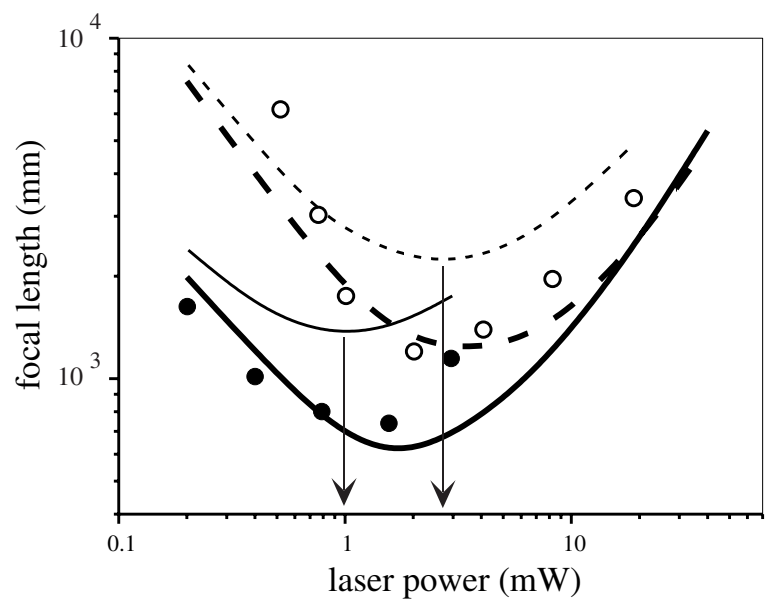

Fig. 4. Electronic nonlinearity: focal length versus laser power. We plot the evolution with laser power of the equivalent focal length of the medium, inferred from measurements of the far-field angular profiles. The experimental data correspond to light detunings $\delta=+3 \Gamma$ (solid circles) and $\delta=+5 \Gamma$ (open circles). The beam is focussed at $d=13 \mathrm{~cm}$ before the sample with a waist $w_{0}=47 \mu \mathrm{m}$. The probe duration is $2 \mu \mathrm{s}$. The thin lines correspond to the calculation from the thin lens model (Eq. (3)). The bold curves are obtained using a numerical model (see text).

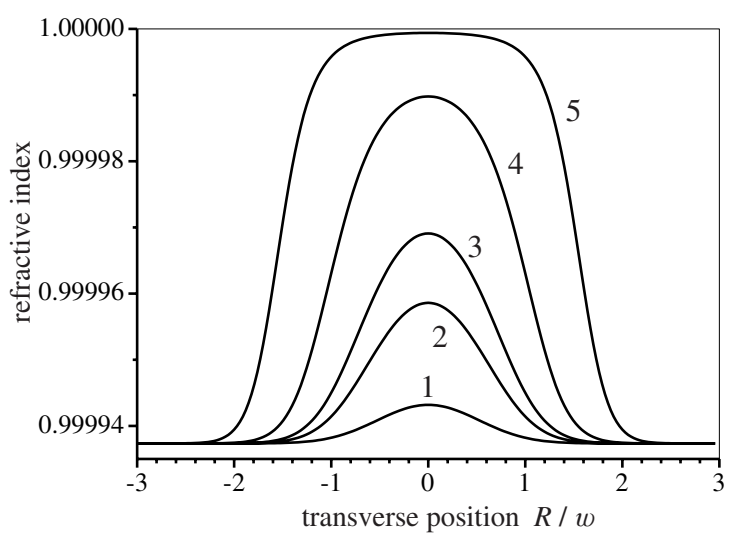

Fig. 5. Transverse profile of refractive index. We plot the transverse variation of the refractive index given by equation (1), for a Gaussian beam of size $w=67 \mu \mathrm{m}$ on the sample, detuning $\delta=+3 \Gamma$, and several on-axis saturation parameter values: (1) $s=0.1$, (2) $s=0.5$, (3) $s=1$, (4) $s=5$ and (5) $s=100 ; s=1$ corresponds to a probe power $P=10 \mu \mathrm{W}$. The atomic density is $\rho=5 \times 10^{10} \mathrm{~cm}^{-3}$.

"saturated" regimes. The Kerr regime is obtained when the saturation parameter on the beam axis $s$ is smaller than unity (curves 1, 2 and 3 in Fig. 5) ; the index profile is then Gaussian and follows the intensity profile. For $s>1$, the electronic nonlinearity saturates and the index profile flattens around the beam axis, yielding a "rectangular" shape for $s \gg 1$ (curve 5 in Fig. 5).

To predict in the simplest possible way the behavior of self-lensing as a function of the various parameters, one can use the following model. Let us assume 
that our system behaves like a thin, ideal (i.e. parabolic) lens whose focal length $f$ is determined by the curvature $c$ of the refractive index around the beam axis: $n(R)=n(0)+c R^{2}+\mathcal{O}\left(R^{3}\right)$. It is quite straightforward to show that this focal length is:

$$
f=\frac{k w^{2}}{4 b_{r e s}} \frac{1+4(\delta / \Gamma)^{2}}{\delta / \Gamma} \frac{(1+s)^{2}}{s}
$$

where $k$ is the wavevector. We recall that the saturation parameter $s$ in the equation above is also a function of $\delta$.

Generally speaking, a short focal length (strong NL effect) is obtained for a large optical thickness and a small beam size. However, expression (3) also shows that there is an optimal laser power for a given beam size and detuning. Indeed, at low saturation $f$ decreases sharply (as $1 / s$ ) when $s$ increases, it reaches a minimum for $s=1$ and then increases linearly for $s \gg 1$. The shortest focal lens for a given beam radius on sample $w$ is obtained by adjusting the intensity and detuning such that $\delta=\Gamma / 2$ and $s=1$. For $w=50 \mu \mathrm{m}$ and $b_{\text {res }}=20$ (parameters close to those of Fig. 3) one obtains $f=4 \mathrm{~mm}$. Since such a short focal length is of the order of the size of the atomic cloud, we expect the "thin lens" model to fail in this regime, where the full propagation inside the nonlinear medium should be considered.

On the other hand, for the large beam size of Figure 4 the focal length is much larger than the sample thickness and the thin lens model should apply. The thin curves in Figure 4 correspond to the prediction of equation (3), without adjustable parameters (solid lines: $\delta=+3 \Gamma$, dashed lines: $\delta=+5 \Gamma$ ). As can be seen, the qualitative agreement is quite good. However, note that the measured focal lengths are generally smaller than the predictions.

Indeed, besides relying on the hypothesis of a thin lens, this simple model neglects two ingredients. First, the lens imprinted in the medium is not parabolic even in the Kerr regime (see Fig. 5). The model above neglects the aberrations introduced by the non-parabolicity of the index profile. Second, this model neglects absorption. The presence of absorption may have two different impacts: first, an inhomogeneous transverse absorption profile leads to diffraction in the far-field; second, absorption introduces an attenuation of the laser intensity as it propagates inside the sample, which in turn affects the refractive index profile.

In reference [26], we have employed a numerical model which included these ingredients (non ideal lens and absorption). We computed the complex amplitude of the field at the output surface of the sample of thickness $L$ :

$$
E(R, z=L)=E(R, z=0) t_{a}(R)
$$

where $t_{a}$ was the amplitude transmission coefficient. The squared modulus of the Fourier transform of $E(R, L)$ yielded the far-field intensity profile of the transmitted beam in the "thin-medium" approximation. However, the transmission coefficient used in reference [26] assumed a small absorption and neglected the impact of the attenuation of the light on the refractive index. In the present

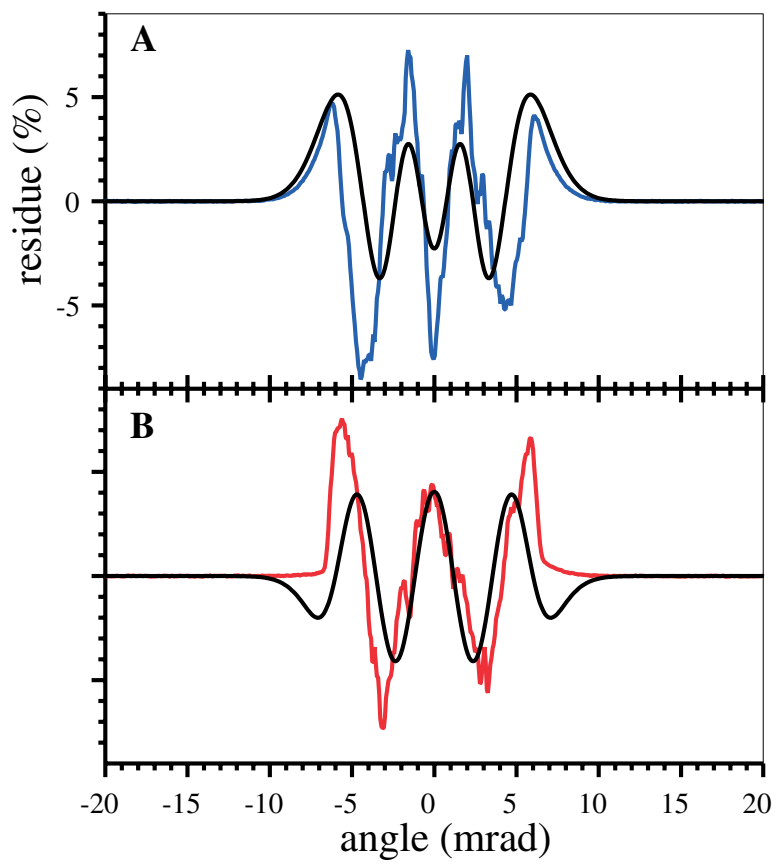

Fig. 6. (Color online) Aberrations of the nonlinear lens. We fit the transmitted beam far-field profile to a Gaussian and plot the residue (noisy curves, in color online) for $\delta=+3 \Gamma$ (A, blue online) and $\delta=-3 \Gamma$ ( $\mathrm{B}$, red online). The laser power is $1.57 \mathrm{~mW}$, the other parameters are as in Figure 4. The smooth curves are obtained using the numerical model based on equation (4) (scaling $\times 5)$.

experiments, this absorption can reach large values and may play a non-negligible role. A more rigorous but timeconsuming approach would require a numerical solution of equation (2) to obtain the full intensity distribution $I(R, z)$. This intensity distribution could then be used to compute the phase distribution of the beam (wavefront) at the output of the sample. In the present paper, we have employed the following approximation. We computed the transmitted intensity $I(R, L)$ by integrating equation (2) and then replaced in equation (1) the incident intensity $I(R)$ by the mean longitudinal intensity inside the medium $[I(R)+I(R, L)] / 2$. Since the relationship between index and intensity is nonlinear, this is clearly not the average index of refraction. However, this quantity includes in a simple (and hopefully not too incorrect) way the information that the light is attenuated inside the sample. The bold curves in Figure 4 are obtained using this numerical model, and are clearly in better agreement with the experimental data than the ideal lens model.

Going one step further, we now look at the deformation of the beam as it interacts with the atomic vapor. This is illustrated in Figure 6, where we plot the residue of the fit of the far-field profile to a Gaussian (i.e. the difference profile-fit), for $\delta=+3 \Gamma$ (Fig. $6 \mathrm{~A}$ ) and $\delta=-3 \Gamma$ (Fig. 6B). The qualitative behavior of the observed deformation is nicely reproduced by the numerical model based on equation (4) (bold lines), although the measured effect is somewhat larger than expected from the model. 
The magnitude of these deformations was found to be very sensitive to the parameters in the simulations, and a quantitative agreement is clearly beyond the reach of this oversimplified model. However, the model confirms that these deformations are due to the non-parabolic shape of the effective lens. We stress that a full quantitative comparison between theory and experiment would require the inclusion of other ingredients such as e.g. the finite thickness of the atomic cloud (which is not negligible compared to the Rayleigh length of the input beam for some of the results presented in this paper) or its non-uniform longitudinal density profile.

\subsection{Beyond the electronic nonlinearity}

As emphasized in Figure 3, the electronic nonlinearity discussed in the previous section does not account for the rather rich behavior observed as the duration of the atomlight interaction is increased. In the following sections, we will analyze the mechanisms responsible for this behavior, namely the radiation pressure and hyperfine pumping. To this end, we will employ a most simple model based only on the photon scattering rate by two-level atoms. As will be shown, this is sufficient to qualitatively reproduce the experimental observations.

\subsubsection{Radiation pressure}

The atoms interacting with the focussed laser beam are subjected to two different forces: the radiation pressure force and the dipole force. The dipole force is associated to the intensity gradient of the laser beam. This gradient is strongest in the transverse dimensions and quite negligible in the longitudinal direction of propagation. The dipole force attracts the atoms towards the beam axis for $\delta<0$ and expels them from the beam for $\delta>0$. With our typical parameters, as e.g. in Figure 3, the dipole potential barrier height is larger than the thermal energy of the cold atoms so that its influence can not in principle be neglected. However, the oscillation period in the potential well is of several ms, larger than the interaction time between the atoms and the beam. We will thus neglect this force in the following.

On the other hand, the radiation pressure induces a momentum transfer along the direction of propagation at a rate:

$$
F_{r p}=\frac{\Gamma}{2} \frac{I(R) / I_{s a t}}{1+4(\delta / \Gamma)^{2}+I(R) / I_{s a t}} m v_{r e c}
$$

where $v_{r e c}=6 \mathrm{~mm} / \mathrm{s}$ is the recoil velocity associated with the absorption of one laser photon, and $m$ the mass of a rubidium atom. This force pushes the atoms along the direction of propagation, thus the spatial displacement of the atoms has no impact at first order on the properties of the medium. However, the atoms initially almost at rest are accelerated and their longitudinal velocity increases with interaction time. Because of the associated Doppler

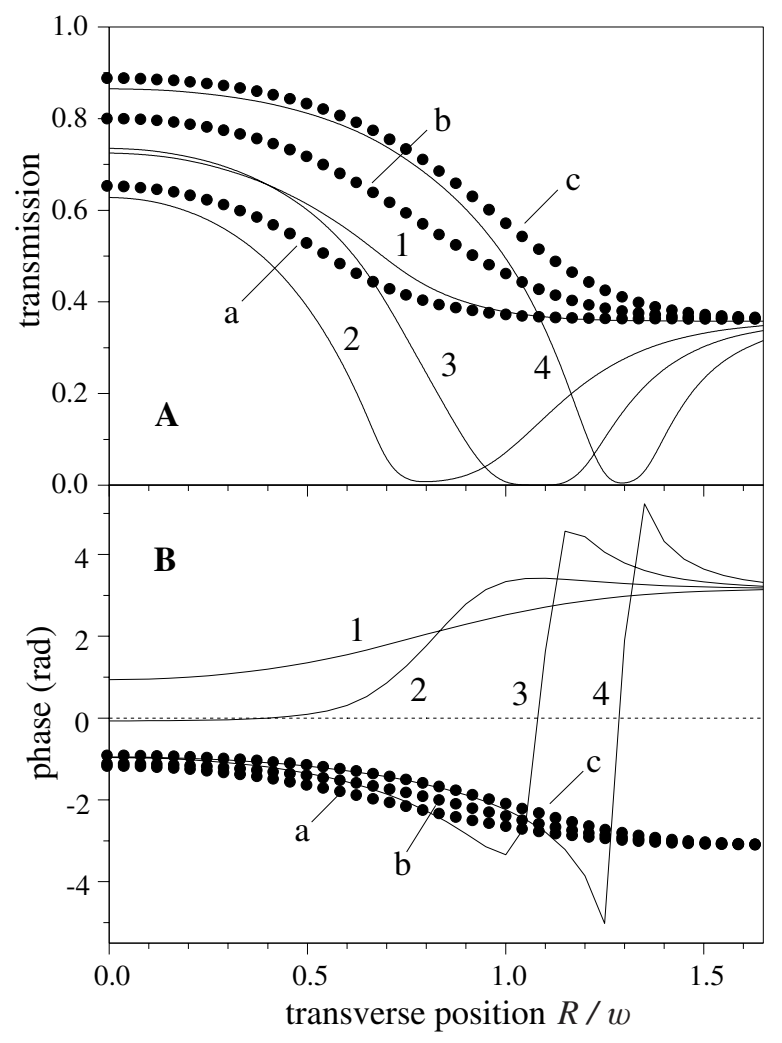

Fig. 7. Impact of radiation pressure (simulation). We simulate (see text) the time evolution of the transverse profiles of phase (reflecting the variations of refractive index) and transmission at the output of the sample, in the absence of hyperfine pumping. The horizontal coordinate is the scaled distance from beam axis $R / w$. The solid curves on panel A show the transmission profiles for $\delta=+3.08 \Gamma: t=0$ (1), $t=150 \mu \mathrm{s}(2)$, $t=300 \mu \mathrm{s}(3)$ and $t=600 \mu \mathrm{s}(4)$. The symbols correspond to $\delta=-3.08 \Gamma: t=0$ (a), $t=200 \mu \mathrm{s}$ (b) and $t=600 \mu \mathrm{s}$ (c). In panel B we plot the phase shift experienced by the transmitted beam.

effect, the laser detuning in the accelerated atomic reference frame is also varying with time. This is a rather fast phenomenon: to change the effective detuning by a quantity $\Gamma$ requires the scattering of $N=\Gamma /\left(k v_{r e c}\right) \approx 800$ laser photons, which takes roughly $80 \mu$ s at a saturation $s=1$. Thus, the effect of radiation pressure is to introduce a time-dependent transverse distribution of effective light frequency (while hyperfine pumping induces a timedependent transverse density profile, see next section). Because the atomic response is highly frequency-dependent, this enables some quite radical transient phenomena as illustrated in Figure 7.

To model the effect of radiation pressure in NL lensing, we use the following procedure. We numerically solve the equation for the atomic motion in the longitudinal direction $z$, as a function of the transverse distance $R$ from the beam axis. We assume that there is no transverse motion of the atoms, which could originate from either the thermal distribution or the dipole force. Knowing the time evolution of the atomic longitudinal velocities, we have 
access to the time-resolved transverse distribution of effective detuning. This allows us to compute the transverse distributions of refractive index and absorption using expressions (1) and (2), which are fed to a Fourier transform calculation to obtain the far-field intensity distribution.

Figure 7 shows some simulated transverse profiles of both the transmission and phase at the output of the sample, as obtained from equations (2) and (1). We recall that the phase shown in Figure 7B is proportional to the difference $n-1$, and thus reflects the profile of refractive index. We plot these profiles for opposite laser detunings $+3.08 \Gamma$ (lines) and $-3.08 \Gamma$ (symbols), and for different interaction times. For these simulations, the optical thickness is $b_{\text {res }}=40$ which yields the small-signal transmission of 0.36 observed in the wings of the beam in Figure 7A. The laser power is $21 \mathrm{~mW}$ and the beam waist of $11 \mu \mathrm{m}$ is positioned at $d=2.75 \mathrm{~mm}$ before the cloud for $\delta>0$ and $d=3 \mathrm{~mm}$ after for $\delta<0$. This small difference of $d$ 's is motivated by the comparison with the experiment (see Fig. 8).

For $\delta<0$, the time evolution of both transmission and phase profiles is rather smooth, without marked deformation. This is due to the fact that the effective detuning is gradually increasing in absolute value while remaining negative. Accordingly, the transmission near the beam axis increases, faster than in the wings where fewer photons are exchanged. The distribution of refractive index evolves very slowly, and is not very different from the initial one even after $600 \mu \mathrm{s}$ (curve c in Fig. 7).

On the contrary, for $\delta>0$ we observe a very strong deformation of the index distribution: atoms near the beam axis rapidly see their effective detuning coming close to resonance (curve 2). Eventually, in an area around the beam axis both the effective detuning and the index curvature change sign (curves 3,4). For long enough interactions (here $t>200 \mu \mathrm{s}$ ), the index profile of the atomic vapor resembles that of a step-index optical fiber with an inner core of higher index ( $n>1$, retarded negative phase) surrounded by a cladding layer of lower index $(n<1$, advanced positive phase). Note that the boundary between the two zones can become very steep $(\simeq 1 \mu \mathrm{m})$ as time passes, at least within the framework of this model. We also stress that the index contrast created in this way is larger than the one obtained with the electronic nonlinearity alone, because of the opposite signs of the effective detuning in the core region and the beam wings. Thus, one consequence of radiation pressure may be the transient establishment of a highly guiding structure inside the sample. This evolution of the refractive index is accompanied by a modification of the transmission which rapidly turns into a bell-shaped curve of high transmission near the axis, surrounded by a "dark" ring whose diameter expands with time. The pronounced minimum of transmission corresponds to atoms near resonance, but located far enough from the beam axis so that the intensity is small, thus yielding a saturation parameter too small to bleach the medium $(s \ll b)$. The position of this minimum also corresponds to the steep boundary of the index profile.

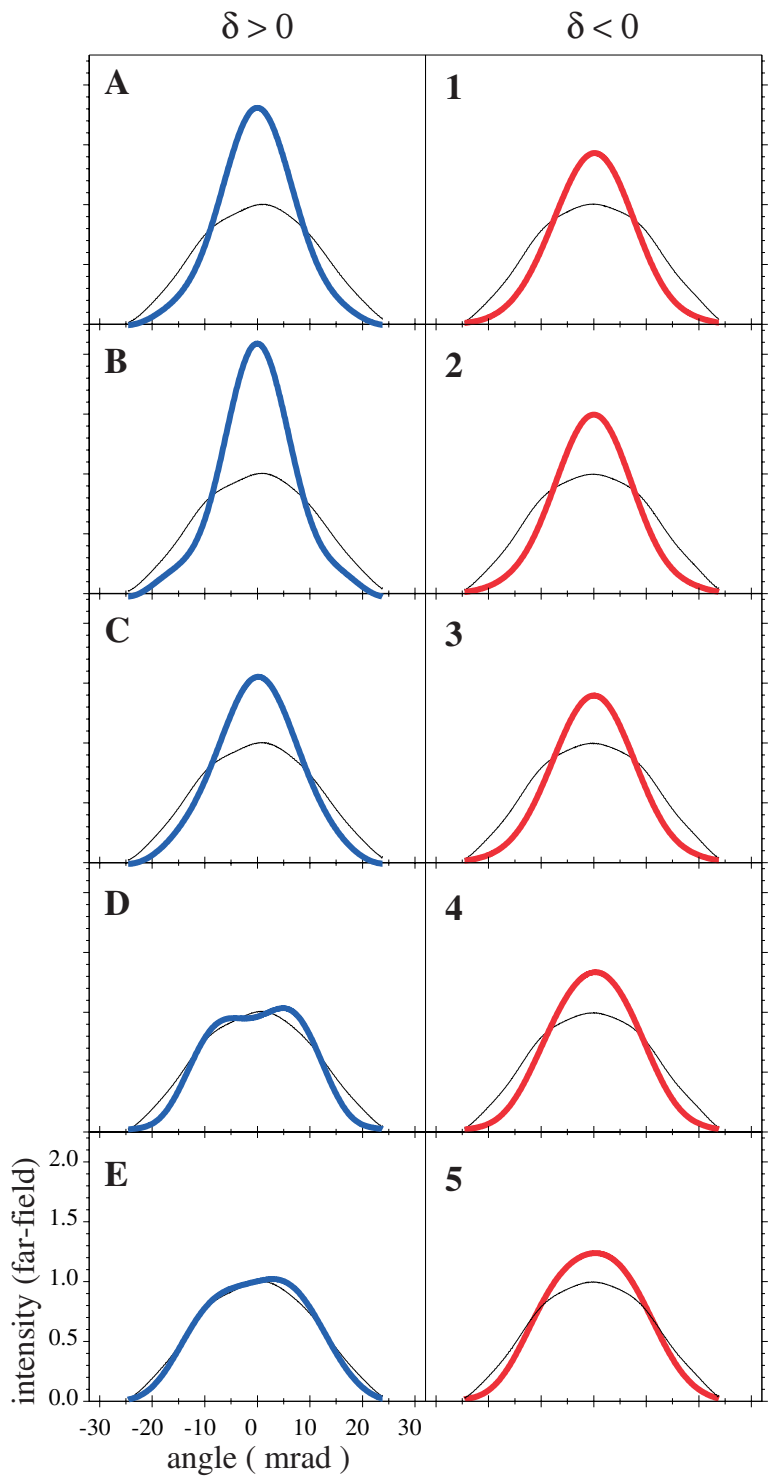

Fig. 8. (Color online) Dynamics of the far-field intensity distribution in the presence of repumper (experiment). We plot cross-sections of the measured far-field intensity distribution with (bold curves, color online) and without (thin curves) cold atoms. The transmission images are recorded in the presence of a repumping laser to suppress hyperfine pumping. The experimental parameters are given in the text. The left column corresponds to a positive detuning (blue online): $\Delta t=0.2 \mu \mathrm{s}$ (A), $\Delta t=60 \mu \mathrm{s}$ (B), $\Delta t=120 \mu \mathrm{s}(\mathrm{C}), \Delta t=200 \mu \mathrm{s}$ (D) and $\Delta t=500 \mu \mathrm{s}(\mathrm{E})$. The right column corresponds to a negative detuning (red online): $\Delta t=0.2 \mu \mathrm{s}$ (1), $\Delta t=40 \mu \mathrm{s}(2)$, $\Delta t=80 \mu \mathrm{s}(3), \Delta t=200 \mu \mathrm{s}(4)$ and $\Delta t=500 \mu \mathrm{s}(5)$.

We compare in Figure 8 the behavior of the transmitted far-field intensity distribution for positive (left column) and negative (right column) detuning $(|\delta|=$ $3.08 \Gamma)$. These transmission profiles were recorded with a repumper laser present during the interaction to suppress hyperfine pumping. The laser beam of total power $21 \mu \mathrm{W}$ has a waist $w_{0}=10.9 \mu \mathrm{m}$. The optical thickness is here $b_{r e s}=18$. As for Figure 3, the beam waist position with 
respect to the sample $d$ was adjusted depending on the sign of $\delta$. We attribute the difference of on-axis transmissions at short time for opposite detunings (plots A and 1) to a slight asymmetry in the waist position, the average distance to the MOT center being $d=2.75 \mathrm{~mm}$.

In contrast to the diaphragm transmission technique used in Figure 3, we now have access to the timedependent deformations experienced by the far-field profiles, which provide some information on the transverse distributions of refractive index and absorption. We first remark that the overall behavior confirms the observations of Figure 3. For $\delta<0$, the evolution of the NL signal is very slow. The on-axis enhancement gradually decreases while the transmitted far-field distribution broadens without noticeable deformation. On the contrary, for $\delta>0$ the evolution is abrupt with a rapid increase of the onaxis transmission within the first $60 \mu \mathrm{s}$ (with an associated narrowing of the transmitted profile), followed by a rapid drop of the nonlinear effect which has vanished after $\simeq 200 \mu \mathrm{s}$. We also observe that the transmitted beam profile now experiences some rather strong deformations, as illustrated by the "bi-component" shape in Figure 8B and the on-axis dip of Figure 8D.

Figure 9 shows the time evolution of two quantities extracted from the profiles of Figure 8. Solid and open circles correspond to positive and negative detunings respectively. We plotted in Figure 9A the on-axis transmission, whose behavior is qualitatively similar to that reported in the bottom row of Figure 3. Quadrant B shows the "far-field magnification" obtained by fitting the measured intensity profiles (in the presence of atoms) to a Gaussian and dividing the obtained size by that of the incident beam. For Gaussian beams, this parameter can readily be used to compute the NL focal length as done in Figure 4. In substantial agreement with the on-axis transmission, we observe very different behaviors depending on the sign of the detuning: for $\delta<0$ the magnification increases quite monotonically towards unity, with a long time constant of several hundreds of $\mu \mathrm{s}$. For $\delta>0$ it first decreases to a marked minimum around $t=60 \mu \mathrm{s}$, corresponding to an equivalent focal length $f \approx 7 \mathrm{~mm}$, then increases sharply towards unity within $200 \mu \mathrm{s}$.

We also reported in Figure 9B the simulated evolution of the far-field magnification (lines). The solid and dashed curves correspond to positive and negative detuning values, respectively. Apart from some quantitative differences, the overall features for both signs of the detuning are well reproduced. We are therefore confident that our simulation captures the main physical ingredient for the beam reshaping in this situation.

This simulation also yielded the profiles shown in Figure 7 , which are helpful in understanding the features of the far-field transmission. For instance, the initial decrease in magnification for $\delta>0$ in Figure 9B is linked to an increasing curvature of the refractive index: atoms near the axis see a smaller effective light detuning, which reduces the refractive index (see Fig. 7). In this initial regime, the index profile remains rather close to a Gaussian and the deformations of the far-field intensity are small. The ef-

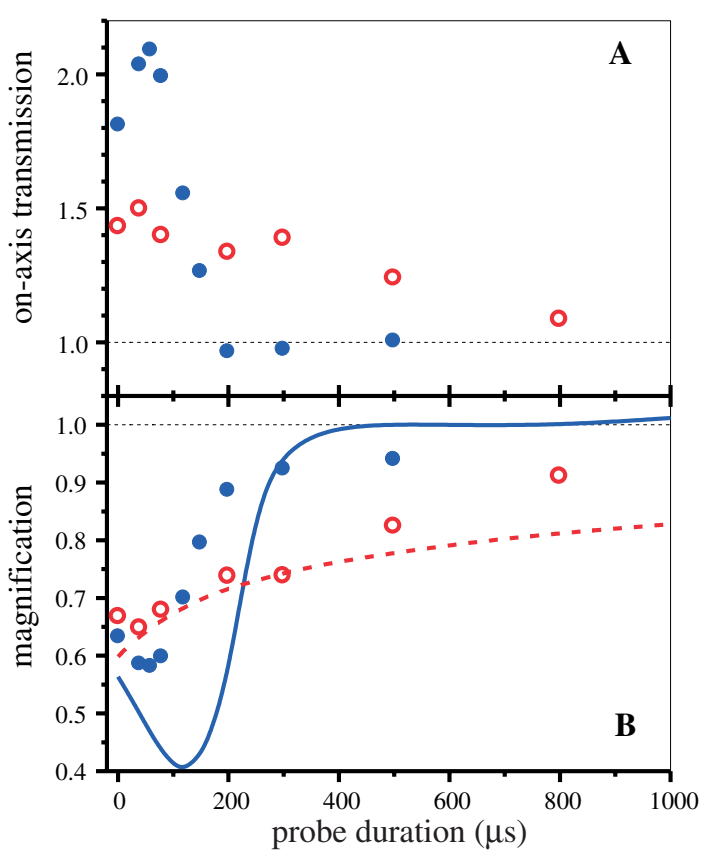

Fig. 9. (Color online) Role of radiation pressure. We plot (symbols) the time evolution of the on-axis transmission (A) and far-field magnification (B, see text) obtained from the experimental transmission profiles of Figure 8. Solid circles correspond to $\delta=+3.08 \Gamma$ (blue online) and open circles to $\delta=-3.08 \Gamma$ (red online). The lines in $\mathrm{B}$ correspond to the results of a numerical simulation (see text), for both positive (solid line, blue online) and negative (dashed line, red online) detunings. Despite some quantitative discrepancies, the simulation reproduces well the asymmetric behavior with respect to detuning which is a signature of radiation pressure.

fect of absorption is here essentially an overall decrease of the transmitted intensity, and thus of the on-axis transmission $T_{0}$. The abrupt increase in magnification towards unity observed for $\Delta t>120 \mu$ s corresponds roughly to the transition to the waveguide-like, rectangular index profile. There, the transmitted beam experiences strong deformations: in the absence of absorption, the steep boundary in the refractive index profiles of Figure 7 would lead to strong oscillations in the far-field. However, these features are smeared out by the strong absorption region which is precisely located at the boundary and masks the effect of the index jump. In the actual experiment, other effects may contribute to reduce the sharpness of the index boundary, such as the transverse atomic motion or heating.

It should be mentioned that, although our numerical simulations are in quite good agreement with the overall features of the experimental data (see Fig. 9B), we are unable to reproduce precisely the profiles of Figures 8 and 11. This could probably be improved by including in the model the finite size of the atomic cloud, i.e. going beyond the thin medium approximation. 


\subsubsection{Hyperfine pumping}

As discussed in Section 2, the coupling of the rubidium atoms with the laser beam in the absence of repumping light results in optical pumping into the lowest $F=2$ hyperfine ground state. Since atoms in this ground state are not sensitive to the laser light, the impact of hyperfine pumping is a decrease of the effective density of the atomic cloud. The rate of optical pumping depends on the laser detuning and on the local light intensity:

$$
\Gamma_{\text {pump }}=\frac{\Gamma}{2}\left(\frac{28}{63}\right) \frac{I(R) / I_{\text {sat }}}{1+4\left(\Delta_{34}+\delta / \Gamma\right)^{2}+I(R) / I_{\text {sat }}}
$$

where $\Delta_{34} \simeq 20 \Gamma$ is the angular frequency separation between the $F^{\prime}=3$ and $F^{\prime}=4$ excited states. In this expression, we neglected the probability of exciting the $F=3 \rightarrow F^{\prime}=2$ transition which is even further detuned than the $F=3 \rightarrow F^{\prime}=3$. The $28 / 63$ factor is due to the decay branching ratio from the $F^{\prime}=3$ state. For a detuning of $+3.08 \Gamma$ or $-3.08 \Gamma$ and the on-axis intensities used in the simulations of the previous section, one obtains a hyperfine pumping time constant $\Gamma_{\text {pump }}^{-1}=2.6$ or $2 \mu$ s respectively. However, because of the transverse intensity profile of the Gaussian beam, the typical decay time of the atomic population in the beam (and in $F=3$ ) can be typically 10 times larger. Still, these time scales are shorter than those associated with radiation pressure. Thus, in a situation where the repumping beam is absent, we expect the NL signal to be dominated by hyperfine pumping even though the effect of radiation pressure is also present.

Indeed, after a short interaction time the transverse density profile of the cloud, which could initially be assumed homogeneous on the scale of the laser profile, will become inhomogeneous with a depleted area near the beam axis and a nearly intact region far in the wings. This process is illustrated by the simulated profiles in Figure 10.

The parameters are identical to those used for Figure 7, except for the repumping laser, now absent. As can be seen, within $40 \mu$ s most atoms inside a radius $w$ have been optically pumped, yielding both a transmission coefficient and a refractive index close to unity. Due to this fast depletion, the specific features of Figure 7 due to radiation pressure are washed out, and the behaviors are now rather similar for positive and negative detunings.

Figure 11 shows how the measured far-field intensity distribution evolves in the presence of hyperfine pumping. We now observe a rather similar behavior for positive and negative detunings, in contrast to the case of Figure 8. Most of the NL lensing signal has vanished after $\Delta t=$ $100 \mu \mathrm{s}$, whereas some deformations of the beam can still be observed, see e.g. panel 5 .

Figure 12 shows the same quantities as in Figure 9 (note however the log scale for the horizontal axis), as extracted from the profiles of Figure 11. The experimental conditions are the same as in Figure 9, but the repumping laser is absent. As expected from the discussion above, the observed behavior depends here little on the sign of the detuning, and the typical time scale for the evolution of the NL lensing signal is now shorter.

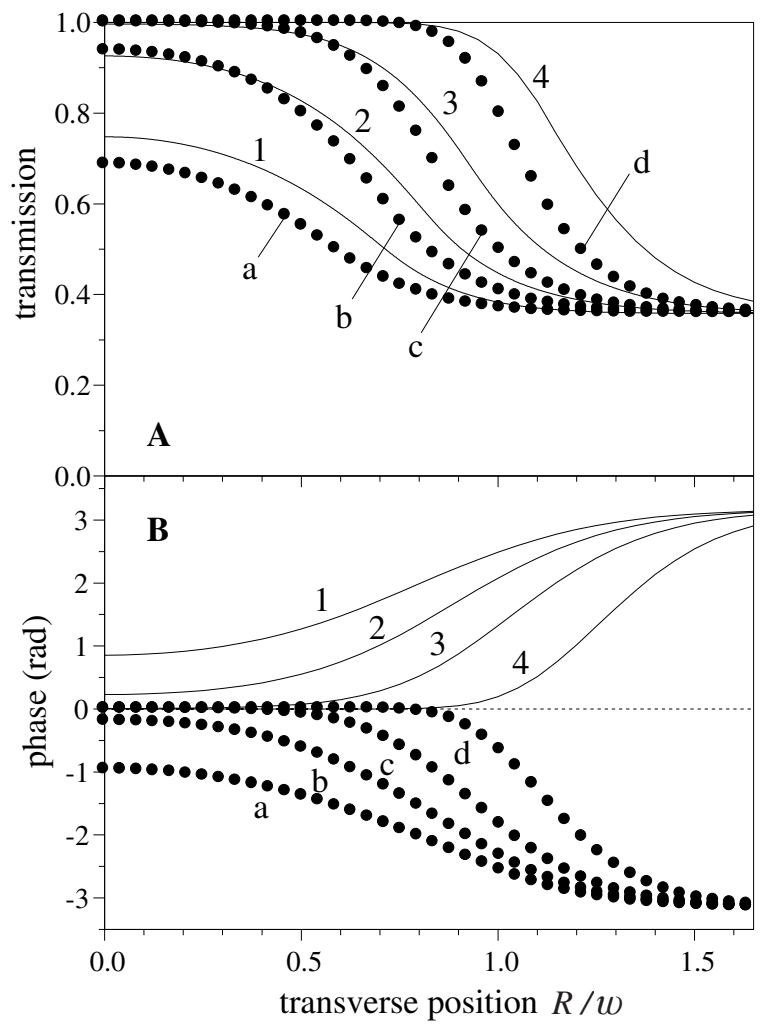

Fig. 10. Impact of hyperfine pumping (simulation). We numerically simulate the time evolution of the transverse profiles of transmission and phase in the conditions of Figure 7, but in the presence of hyperfine pumping (no repumping laser). The lines and symbols correspond to $\delta=+3.08 \Gamma$ and $-3.08 \Gamma$ respectively: $\Delta t=0.2 \mu \mathrm{s}$ (1 and a), $\Delta t=3 \mu \mathrm{s}(2$ and $\mathrm{b})$, $\Delta t=10 \mu \mathrm{s}(3$ and $\mathrm{c})$, and $\Delta t=40 \mu \mathrm{s}(4$ and $\mathrm{d})$. The observed behavior is now nearly symmetric with respect to the sign of detuning, indicating that hyperfine pumping dominates over the Doppler shift associated with radiation pressure.

As in the previous section, we can use the transverse profiles of transmission and phase (see Fig. 10) to compute the temporal evolution of e.g. the far-field magnification. This yields the lines in Figure 12B, where the parameters are identical to those of Figure 9B but without repumping laser. As can be seen, the obtained behavior is qualitatively quite similar to the experimental one, albeit with a significantly shorter time constant. The initial phase of decreasing magnification is associated to an increase of the transverse gradient of refractive index (via a curvature of the transverse density profile), while the index profile remains relatively Gaussian-like. In other words, hyperfine pumping in itself creates a lens whose effects adds to that of the electronic nonlinearity.

The following phase of vanishing NL signal corresponds to the establishment of a rather flat index profile over a large central portion of the laser beam, surrounded by a different index in the wings of the beam. In this situation where the radial cross-section of the index profile is roughly rectangular, the NL lensing disappears when the boundary between the regions of different index is located 


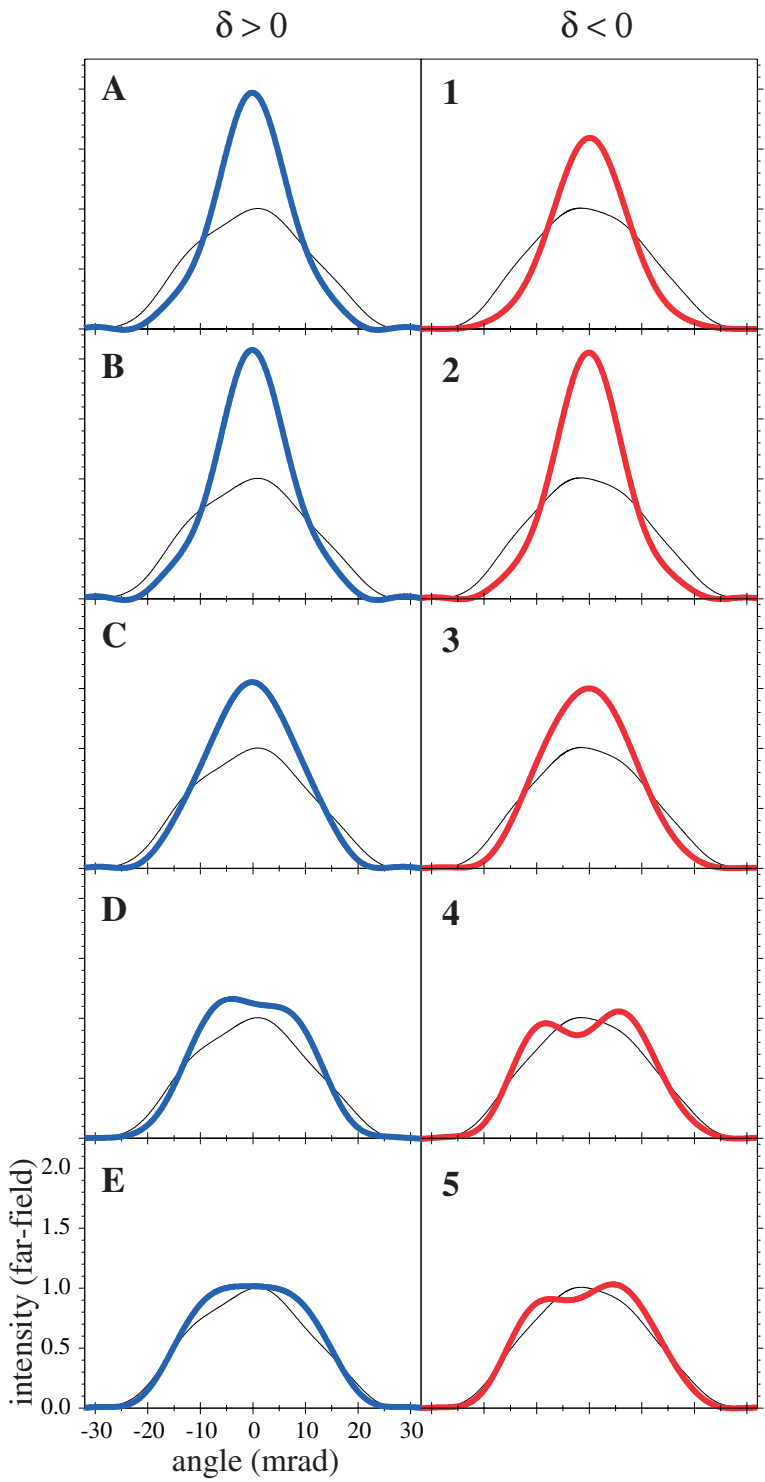

Fig. 11. (Color online) Dynamics of the far-field intensity distribution (no repumper). Same as Figure 8, but without repumping laser. The left column corresponds to $\delta>0$ (blue online) while the right column is obtained for $\delta<0$ (red online). The interaction times are: $\Delta t=2 \mu \mathrm{s}$ (A and 1), $\Delta t=10 \mu \mathrm{s}$ (B and 2), $\Delta t=40 \mu \mathrm{s}$ (C and 3), $\Delta t=100 \mu \mathrm{s}$ (D and 4$)$ and $\Delta t=260 \mu \mathrm{s}(\mathrm{E}$ and 5$)$.

at a distance $R \geq w$ from the beam axis. Qualitatively, the evolution is similar to the case of positive detuning in the absence of hyperfine pumping (see Fig. 9B, solid line): an initial decrease followed by a fast increase towards unity, albeit with different magnitude and time constants. In the two situations (without and with optical pumping), the physical mechanism is different: in the earlier the index variation is driven by the effective detuning, while in the latter it is the effective density which dominates. The case of $\delta<0$ and no hyperfine pumping is qualitatively different, because the refractive index profile is experiencing little deformation and remains almost Gaussian. There,

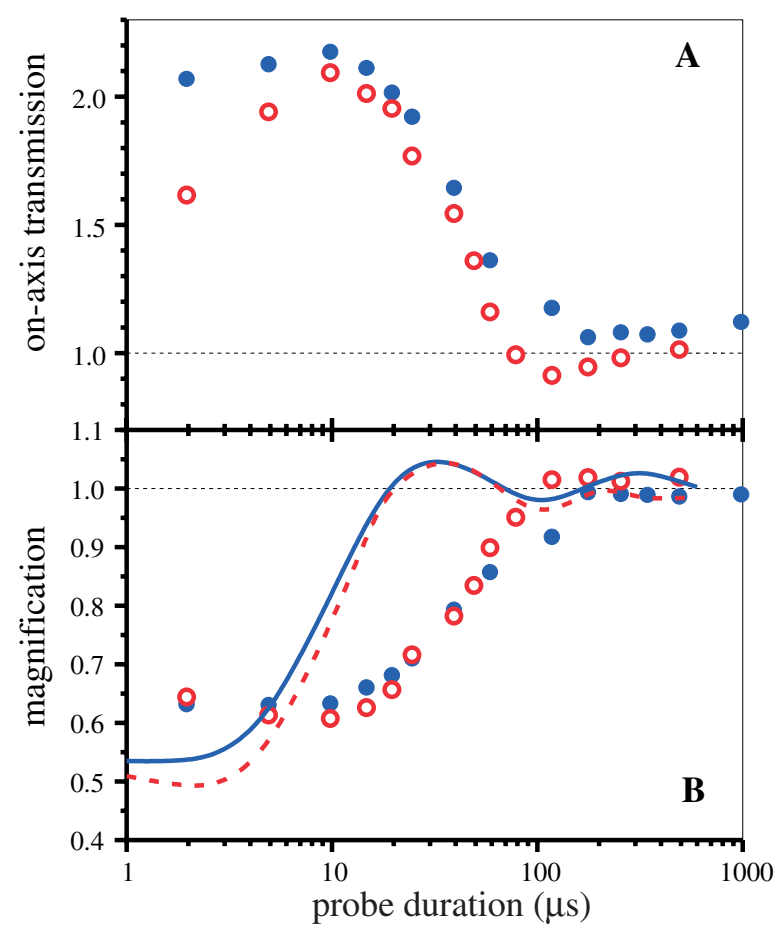

Fig. 12. (Color online) Role of hyperfine pumping. As in Figure 9 , we plot the time evolution of the on-axis transmission (A) and far-field magnification (B) obtained from the experimental transmission profiles of Figure 11, in the absence of the repumping laser. Solid circles correspond to $\delta=+3.08 \Gamma$ (blue online) and open circles to $\delta=-3.08 \Gamma$ (red online). The lines in $\mathrm{B}$ correspond to the results of the numerical simulation for both positive (solid line, blue online) and negative (dashed line, red online) detunings. The parameters of the simulation are identical to those of Figure 9B, but without repumping laser.

the "spherical lens" analysis of Section 3.1 applies and the slow evolution of the far-field magnification can be understood in terms of a decreasing curvature of the refractive index profile alone.

\section{Conclusion}

In this paper we reported a time-resolved study of selflensing of a laser beam by a cloud of cold rubidium atoms. The analysis of the experimental data shows the existence of four distinct nonlinear mechanisms (electronic, Zeeman pumping, radiation pressure and hyperfine pumping) with different time scales. This offers the opportunity to manipulate (to some extent) the $\chi^{3}$ of the nonlinear medium simply by selecting the appropriate interaction time. The experimental data are in good qualitative agreement with a simple model which allow us to understand the underlying mechanisms. In particular, our simulations show that the resonant radiation pressure force exerted by the laser can establish in transient a highly guiding structure which could be used to obtain e.g. "soliton waves" in the cold atomic vapor. Indeed, the coupling of the mechanical 
aspect of light-matter interaction to the traditional nonlinear optics is one intriguing new feature of nonlinear optics in samples of cold atoms. Further investigations in this line of research will include the search for solitons and pattern formation in the presence of optical feedback. Besides the need to either counteract or utilize hyperfine pumping, our results indicate that mechanical effects in the longitudinal direction set in after several tens of $\mu$ s. Since preliminary estimates place the time to form transverse density gratings in the $100 \mu$ s to some milliseconds range, this provides some further support for the suggestion in [25] to leave the cooling beams on during a pattern formation experiment.

We thank the CNRS and the PACA Region for financial support. T.A. is grateful to the Alexander-von-Humboldt Foundation for a travel grant.

\section{References}

1. M. Hercher, J. Opt. Soc. Am. 54, 563 (1964)

2. P.L. Kelley, Phys. Rev. Lett. 15, 1005 (1965)

3. J.E. Bjorkholm, A. Ashkin, Phys. Rev. Lett. 32, 129 (1974)

4. Y.R. Shen, Prog. Quantum Electron. 4, 2 (1975)

5. J. Marburger, Prog. Quantum Electron. 4, 35 (1975)

6. R.W. Hellwarth, J. Opt. Soc. Am. 67, 1 (1977)

7. A. Yariv, D.M. Pepper, Opt. Lett. 1, 16 (1977)

8. D.M. Bloom, G.C. Bjorklund, Appl. Phys. Lett. 31, 592 (1977)

9. R.G. Brewer, Phys. Rev. Lett. 19, 8 (1967)

10. F. Shimizu, Phys. Rev. Lett. 19, 1097 (1967)

11. A.C. Cheung, D.M. Rank, R.Y. Chiao, C.H. Townes, Phys. Rev. Lett. 20, 786 (1968)

12. R.S. Benning, V. Wong, D.M. Marino, D.L. Aronstein, R.W. Boyd, C.R. Stroud Jr, S. Lukishova, D.J. Gauthier, Phys. Rev. Lett. 88, 113901 (2002)

13. A. Gahl, J. Seipenbusch, A. Aumann, M. Möller, W. Lange, Phys. Rev. A 50, R917 (1994)

14. T. Ackemann, Y. Logvin, A. Heuer, W. Lange, Phys. Rev. Lett. 75, 3450 (1995)

15. A.V. Mamaev, M. Saffman, A.A. Zozulya, Phys. Rev. Lett. 76, 2262 (1996)
16. T. Ackemann, W. Lange, Appl. Phys. B 72, 21 (2001)

17. P. Mandel, M. Tlidi, J. Opt. B: Quantum Semiclass. Opt. 6, R60 (2004)

18. L.A. Lugiato, M. Brambilla, A. Gatti, Adv. A. Mol. Opt. Phys. 40, 229 (1999)

19. F.T. Arecchi, S. Boccaletti, P.L. Ramazza, Phys. Rep. 318, 1 (1999)

20. T. Chanelière, D. Wilkowski, Y. Bidel, R. Kaiser, C. Miniatura, Phys. Rev. E 70, 036602 (2004); S. Balik, P. Kulatunga, C.I. Sukenik, M.D. Havey, D.V. Kupriyanov, I.M. Sokolov, J. Mod. Opt. 52, 2269 (2005)

21. H. Cao, Waves in Random Media 13, R1 (2003); S.C. Rand, C. Soukoulis, D.S. Wiersma, J. Opt. Soc. Am. B 21, 98 (2004)

22. B.A. Malomed, D. Mihalache, F. Wise, L. Torner, J. Opt. B: Quantum Semiclass. Opt. 7, R53 (2005)

23. T. Ackemann, A. Aumann, E. Große Westhoff, Yu. A. Logvin, W. Lange, J. Opt. B: Quantum Semiclass. Opt. 3, S124 (2001)

24. M. Saffman, Phys. Rev. Lett. 81, 65 (1998)

25. G.A. Muradyan, Y. Wang, W. Williams, M. Saffman, Absolute instability and pattern formation in cold atomic vapors, in Nonlinear Guided Waves and Their Applications (Dresden, 2005), Paper ThB29

26. G. Labeyrie, T. Ackemann, B. Klappauf, M. Pesch, G.-L. Lippi, R. Kaiser Eur. Phys. J. D 22, 473 (2003)

27. Y. Wang, M. Saffman, Phys. Rev. A 70, 013801 (2004)

28. Y. Wang, M. Saffman, Opt. Commun. 241, 513 (2004)

29. G.L. Gattobigio, F. Michaud, J. Javaloyes, J.W.R. Tabosa, R. Kaiser, Phys. Rev. A 74, 043407 (2006)

30. D. Kruse, C. von Cube, C. Zimmermann, Ph.W. Courteille, Phys. Rev. Lett. 91, 183601 (2003)

31. J.E. Bjorkholm, A. Ashkin, Phys. Rev. Lett. 32, 129 (1974)

32. M. Sheik-Bahae, A. Said, E.W.V. Stryland, Opt. Lett. 14, 955 (1989)

33. G. Labeyrie, C.A. Müller, D.S. Wiersma, C. Miniatura, R. Kaiser, J. Opt. B: Quantum Semiclassical Opt. 2, 672 (2000)

34. T. Ackemann, T. Scholz, Ch. Vorgerd, J. Nalik, L.M. Hoffer, G.L. Lippi, Opt. Commun. 147, 411 (1998)

35. A time constant of $20 \mu \mathrm{s}$ is estimated for the conditions of reference [27] 